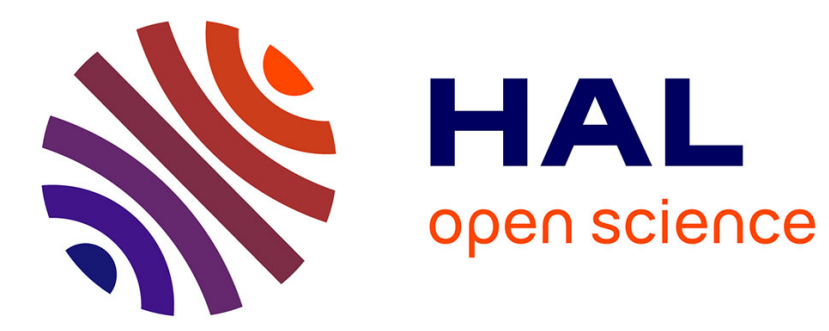

\title{
Surgical Augmented Reality with Topological Changes
}

Christoph Paulus, Nazim Haouchine, David Cazier, Stéphane Cotin

\section{To cite this version:}

Christoph Paulus, Nazim Haouchine, David Cazier, Stéphane Cotin. Surgical Augmented Reality with Topological Changes. MICCAI 2015: Medical Image Computing and Computer-Assisted Intervention, Oct 2015, Munich, Germany. 10.1007/978-3-319-24553-9_51 . hal-03433793

\section{HAL Id: hal-03433793 https://hal.science/hal-03433793}

Submitted on 17 Nov 2021

HAL is a multi-disciplinary open access archive for the deposit and dissemination of scientific research documents, whether they are published or not. The documents may come from teaching and research institutions in France or abroad, or from public or private research centers.
L'archive ouverte pluridisciplinaire HAL, est destinée au dépôt et à la diffusion de documents scientifiques de niveau recherche, publiés ou non, émanant des établissements d'enseignement et de recherche français ou étrangers, des laboratoires publics ou privés. 


\title{
Surgical Augmented Reality with Topological Changes
}

\author{
Christoph J. Paulus ${ }^{12}$, Nazim Haouchine ${ }^{12}$, David Cazier $^{2}$, and Stéphane \\ Cotin $^{12}$ \\ 1 Inria Nancy Grand Est, Villers-les-Nancy, France \\ 2 Université de Strasbourg, ICube Lab, CNRS, Illkirch, France
}

\begin{abstract}
The visualization of internal structures of organs in minimally invasive surgery is an important avenue for improving the perception of the surgeon, or for supporting planning and decision systems. However, current methods dealing with non-rigid augmented reality only provide augmentation when the topology of the organ is not modified. In this paper we solve this shortcoming by introducing a method for physics-based non-rigid augmented reality. Singularities caused by topological changes are detected and propagated to the pre-operative model. This significantly improves the coherence between the actual laparascopic view and the model, and provides added value in terms of navigation and decision making. Our real time augmentation algorithm is assessed on a video showing the cut of a porcine liver's lobe in minimal invasive surgery.
\end{abstract}

\section{Introduction}

In recent decades, considerable advances in the introduction of augmented reality during surgery have been achieved [1]. More particularly, the scientific community and clinicians have been focusing on minimally invasive surgery (MIS). This kind of surgery has gained popularity and become a well-established procedure thanks to its benefits for the patients in term of haemorrhaging risk reduction and shortened recovery time. However, it remains complex from a surgical point of view, mainly because of the reduced field of view which considerably impacts depth perception and surgical navigation.

Recently, there has been a great deal of ongoing research efforts towards automatic registration between pre- and intra-operative data in MIS considering the elastic organ behavior. Patient-specific biomechanical models have demonstrated their relevance for volume deformation, as they allow to account for anisotropic and elastic properties of the shape and to infer in-depth structure motion [2], [3]. In [4], a 4D scan of the heart is jointly used with a biomechanical model to couple the surface motion with external forces derived from camera data. This method uses the cyclic pattern of the heart deformations to improve the registration. A local tuning of the deformation is used to propagate the surface deformation to in-depth invisible structures. In the context of augmented reality for liver surgery, [3] used a heterogeneous model that takes into account 
the vascular network to improve the soft tissue behavior while real-time performance is obtained using adequate mesh resolution and pre-computed solvers. In [2], a physics-based shape matching approach is proposed. Non-rigid registration between the pre-operative elastic model and the intra-operative organ shape is modeled as an electrostatic-elastic problem. The elastic model is electrically charged to slide into an oppositely charged organ shape representation.

Despite such recent improvements in the field of surgical augmented reality, no study has yet investigated the impact of cutting or resection actions performed during the operation. Given that these are essential steps of any surgical procedure, it is obvious that if the meshes of the underlying mechanical model are not correctly modified, significant errors are generated in the registration and consequently in the estimation of internal structures or tumor localization. In the context of image-guided neurosurgery, Ferrant et al. [5] proposed to handle registration issues induced by tumor resection by updating a biomechanical brain model accordingly with topological changes. These changes consist of removing the elements of the brain model that contains the resected tumor and surrounded area.

In the computer graphics domain, there is ongoing work on methods that take account of the mesh updates induced by cutting, fracture or tearing. A comprehensive overview of cuts in soft tissue simulation is provided in [6]. The simulation of surgical cuts raises specific questions. Elastic and in some cases plastic deformations are required for accurate simulations. The surgeon's manipulations may include cuts, cauterization or tearing of the organs. The computations must therefore handle topological changes or updates in the connectivity of the underlying mesh. Handling such mechanical models and mesh operations implies elevated computational costs and makes it challenging to maintain real-time performance, that is required by augmented reality applications. The approach presented in [7] addresses these issues. The method is based on the composite finite element method, that embeds a fine grid into a coarse uniform hexahedral grid. The fine mesh is used for the visualization and collision and the simulation uses the coarse one, so reducing the computation time. Cuts are performed on the fine level grid that stores the separation information. As soon as a complete separation of the fine grid occurs in a coarse element, it is duplicated to represent the cut. Visually pleasing results are obtained in real-time. However, as the elements of the coarse mesh can only be completely cut, the simulation does not react instantly on partial cuts.

The main contributions of this paper are 1) a method to detect a cut in three-dimensional soft structures by analyzing the motion of tracked surface points and 2) an algorithm for applying the detected topological changes to the preoperative model in real-time. This leads to an improved coherence between the actual surgical situation and the (updated) pre-operative data, therefore positively impacting the accuracy of the navigation. 


\section{Method}

In this section we give a short overview of our method. We process the information from a monocular video stream similar to that provided by endoscopic cameras. This video captures the manipulations of a surgeon on the targeted organ on which deformations and cuts are performed with a scissor-grasper or any similar surgical tool. We suppose that a virtual 3D model of the organ is provided and initially registrated to the first frame of the video. Such a model is usually obtained during pre-operative diagnostic operations from some medical imaging techniques. The biomechanical behavior of the virtual organ is modeled using a non-linear elastic deformation law computed using a finite element method (FEM). The real organ, through feature points captured on the video and the virtual organ are coupled in a way that the motions in the videos are reproduced by the virtual organ. After a surgical cut, differences between the motions of the real and virtual organs appear. We detect and analyze those differences to predict the occurrence of a cut. Detected cuts are then reproduced on the virtual organ thus improving the following registration steps.

\subsection{Coupling Real and Virtual Organs}

We rely on the tracking and spatiotemporal registration as described in [8] where feature points acquired from a camera constrain a non-linear elastic model. The visual tracking yields a set of features $\mathcal{F}=\left\{f_{i} \in \mathbb{R}^{2}\right\}$ chosen in the video stream. The virtual organ is represented by a $3 \mathrm{D}$ mesh with vertices in the set $\mathcal{V}=\left\{v_{m} \in \mathbb{R}^{3}\right\}$. Each feature point $f_{i}$ is associated with a virtual feature point $f_{i}^{v} \in \mathbb{R}^{3}$ lying on the boundary surface of $\mathcal{V}$. The points $f_{i}^{v}$ are initialized with the first frame of the video as the intersection of the line of sight from the camera's position to $f_{i}$ with the boundary of $\mathcal{V}$. Each virtual feature is registered in an element of the FEM mesh and expressed as barycentric coordinates of the element's vertices.

In order to compare the positions of the features $f_{i}$ and the corresponding virtual features $f_{i}^{v}$, the points $f_{i}^{v}$ are projected onto the plane of the $f_{i}$, i.e. the $2 \mathrm{D}$ plane of the video in the 3D scene. In the following, we use $\overline{f_{i}^{v}}=P\left(f_{i}^{v}\right)$ to denote those projections, with the projection matrix $P$ of the camera. As the features move in the video, they introduce a stretching energy $W_{S}(\mathcal{F}, \mathcal{V})$ between each feature $f_{i}$ and its projected virtual feature $\overline{f_{i}^{v}}$ :

$$
W_{S}(\mathcal{F}, \mathcal{V})=\sum_{i} \frac{1}{2} k_{i}\left\|f_{i}-\overline{f_{i}^{v}}\right\|^{2}
$$

The parameters $k_{i}$ are experimentally chosen and are of the same order of magnitude as the Young's modulus of the organ. In addition, the biomechanical object is constrained by fixing nodes at predefined positions: $v_{m}=v_{m}^{D}, m \in B$. The internal elastic energy of the virtual organ is $W_{I}(\mathcal{V})=\sum_{e} W_{e}$, accumulating the strain energy $W_{e}$ of the elements related to a Saint Venant-Kirchhoff material. Finally, the deformation of the virtual organ is expressed as a minimization 
problem between internal elastic energy and stretching energy $W_{I}(\mathcal{V})+W_{S}(\mathcal{F}, \mathcal{V})$ with the constraint that $v_{m}=v_{m}^{D}$, for all $m \in B$. The solution of the problem is the updated set of vertices $\mathcal{V}$. The positions of the virtual features $f_{i}^{v}$ are updated using the stored barycentric coordinates and the updated $\mathcal{V}$.

The stretching energy links the virtual features $f_{i}^{v}$ to the real ones $f_{i}$. When the virtual organ correctly follows the motion of the real one, the vector $d_{i}=$ $f_{i}-\overline{f_{i}^{v}}$ changes continuously in the neighborhood of $f_{i}$. This vector encodes simultaneously the Euclidean distance between $f_{i}$ and $\overline{f_{i}^{v}}$ and direction of the relative motion of the organ and its virtual representation. In the next section, the vector $d_{i}$ is used to detect potential cuts in the real organ.

\subsection{Detecting Discontinuities in Motion}

With a continuous deformation of the manipulated organ, the projections of the virtual features $\overline{f_{i}^{v}}$ smoothly follow the tracked points $f_{i}$. When a cut occurs, the motion of $\overline{f_{i}^{v}}$ and $f_{i}$ starts to diverge, because the cut is not represented in the virtual organ. To detect such divergent motions, we analyze the vectors $d_{i}$ of neighboring virtual features. We define the set $\mathcal{N}=\{(i, j)\}$ of neighboring pairs of virtual features such that the Euclidean distance $\left\|\bar{f}_{i}^{v}-\bar{f}_{j}\right\|$ is lower than a given radius $r$ that depends on the detected features. The neighborhood $\mathcal{N}$ is initialized based on the features obtained in the first frame of the video. These notions are illustrated in figure 1.

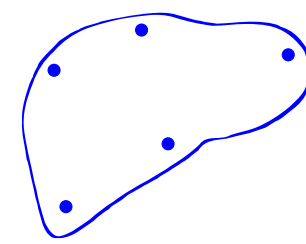

(a)

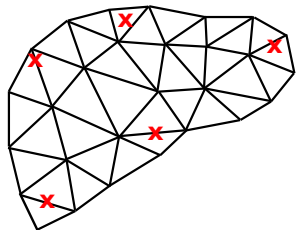

(b)

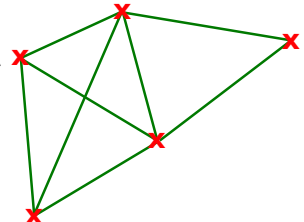

(c)

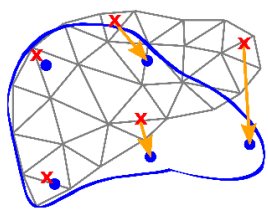

(d)

Fig. 1. (a) Real object with features $f_{i}$ (blue disks); (b) Mesh with the virtual features $\overline{f_{i}^{v}}$ (red crosses); (c) Set $\mathcal{N}$ of neighboring virtual features (neighborhood relations in green); (d) Differences $d_{i}$ between $f_{i}$ and $\overline{f_{i}^{v}}$ (orange vectors)

To evaluate whether a discontinuity occurs in the way $d_{i}$ evolves, we introduce the measure $\mu_{i j}=\left\|d_{i}-d_{j}\right\|$. We calculate the average distance $\bar{\mu}$ of the measures $\mu_{i j}$ over the set $\mathcal{N}$. Discontinuities between two features $f_{i}$ and $f_{j}$ are detected by finding the outliers $\mu_{i j}>\bar{\mu} \epsilon$, with a threshold $\epsilon$ dependent on the scenario. For the moment the monocular camera fixes the tracked features to the plane of the video, thus discontinuities in the motion along the z-axis can not be detected. Intuitively, the outliers correspond to pairs of features $f_{i}, f_{j}$ whose difference vectors $d_{i}, d_{j}$ differ too much - either in length or direction (figure $2(\mathrm{a})$ ). 


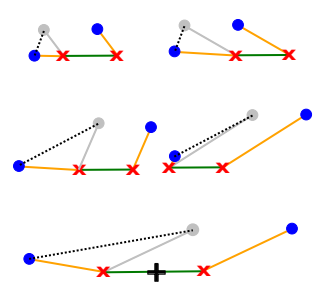

(a)

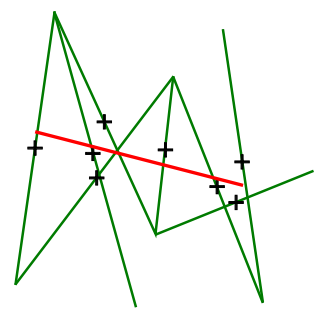

(b)

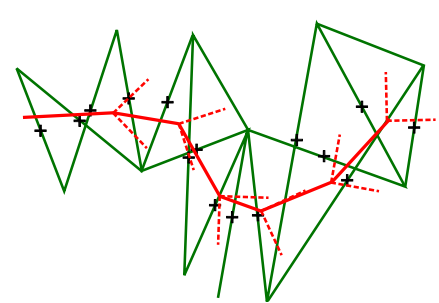

(c)

Fig. 2. (a) Neighbor configurations: dotted lines illustrate computation of measure $\mu_{i j}$, the last configuration introduces a separation point (black cross); (b) Initialization of the cut (red line) averaging the separation points (black crosses) (c) Progression of the cut, with the angular restriction (dashed red lines)

The computed outliers define a region where a cut is likely to occur. To capture the location of this cut, we insert separation points between diverging features near their barycenters. The simulated cut is thus initialized as a line minimizing the Euclidean distance to a fixed number of separation points (figure 2(b)). We assume that the cutting process is continuous, i.e. that the separation path can advance in two directions, starting from the extremities of the initial separation line. Thus, we represent the separation as two lines $l_{1}$ and $l_{2}$, that can move independently from the first initialization line. This sequence of lines defines a separation polygon for each time step (figure 2(c)). The separation polygon is finally extruded along a depth vector to define a separation surface. This vector is either the direction of the camera or a predefined direction.

\subsection{Robust processing of the cut}

To ensure a trustworthy and robust detection of cuts, unrealistically tracked features that jump or slide in improbable directions need to be filtered out. The underlying mechanical model used to deform the virtual organ has the desirable property of regularizing or smoothing the movements of the points $\overline{f_{i}^{v}}$.

Again, we use the difference vector between the positions of neighbors to determine outliers, but this time comparing along the combination of the resolution of time and space. Precisely, we consider the evolution of $d_{i}$ during a time step using the measure $E_{i}=\left\|d_{i}(t+\Delta t)-d_{i}(t)\right\|$ and we determine outliers comparing to the average of this measure. If a feature $f_{i}$ has been identified as an outlier, then it is not used for the detection of the cut.

In addition, the propagation of the cut can be restricted in order to react on noisy data. First, an angular restriction $\alpha$ constrains the lines to only move in the desired direction. Secondly, the length of each line can be adapted, introducing a minimal and a maximal progression for each cut line. Thirdly, new cut lines are only inserted when a sufficient number of separation points have been inserted. 
Those constraints present two advantages: (i) it reduces the wrong detections due to too large motions and (ii) the detail of the separation polygon can be controlled.

The separation is incorporated into the volumetric mesh combining a remeshing approach (similar to [9]) with a simple snapping of the volumetric vertices to the cut [10]. However, the detection method we propose is independent of the separation algorithm, other efficient algorithms like [7] could be used as well.

\section{Experimental Results}

In this section, we demonstrate the potential of our approach to detect a surgical cut from the motion of features $f_{i}$ extracted from a video stream and to replicate the corresponding topological changes on a virtual model $\mathcal{V}$ augmenting the view.

Our algorithm was applied on two scenarios involving highly elastic silicone bands which are cut and then video recorded while being manipulated to induce deformations. The feature tracking, deformable model update, cut detection and topological changes are performed in real-time. The final positions show the advantage of our method over an uncut mesh (see figure 3). This is quantitatively evaluated using a classical dice coefficient on the two dimensional domain of the video data. The results for the first case scenario (object cut on the side) are 0.815 when not accounting for topological changes, and 0.952 when using our algorithm. Results for the second case scenario (object cut in the middle) are 0.900 for the uncut mesh, and 0.964 when applying our method.
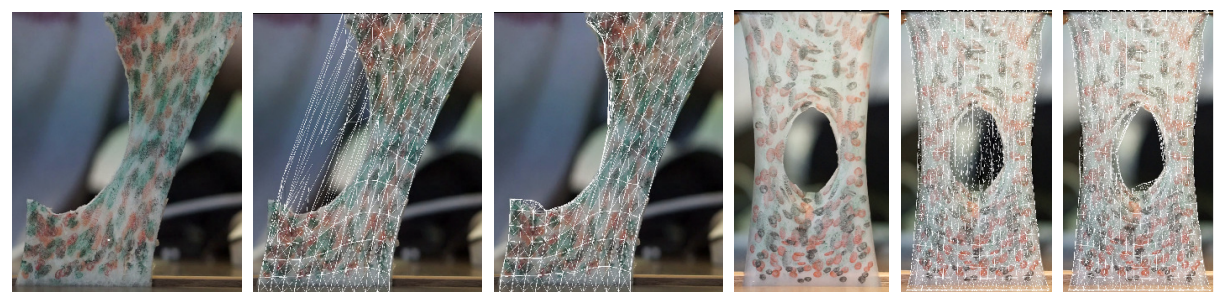

Fig. 3. Examples of a detected cut in silicone, augmented with an uncut/cut model

We then evaluated our approach on a video clip involving a cut being performed on a porcine liver lobe. The initial and the final frames are shown in figures $4(\mathrm{a})$ and $4(\mathrm{~d})$. In this example, the algorithm extracts 438 features $f_{i}$ from the video stream (fig. 4(a)), 32 are identified to be outliers in the advancement of the video. The features deform a volumetric mesh, using the spring energy $W_{S}(\mathcal{F}, \mathcal{V})$. Figure $4(\mathrm{~b})$ illustrates the initial configuration of the vertices $\mathcal{V}$. We calculate the measure $\mu$ on 18503 pairs of features and lose 1478 of these pairs in the course of the simulation due to identified outliers - the initial neighborhood information $\mathcal{N}$ is displayed in figure $4(\mathrm{c})$. 
When applying our method, the measure $\mu_{i j}$ is calculated on the neighboring pairs of features in $\mathcal{N}$ and a pair of features is identified to be cut using a threshold $\epsilon=7.0$. The resulting three-dimensional representation of the liver, as illustrated in figures $4(\mathrm{f})$ and $(\mathrm{g})$, is very similar to the actual organ shape.

To analyze our results, we compute the dice measure comparing the surfaces of the uncut pre-operative mesh and the cut mesh obtained with our method. The dice coefficient associated with our result is 0.963 , whereas it was 0.906 for the uncut object, confirming the benefits of the proposed method.

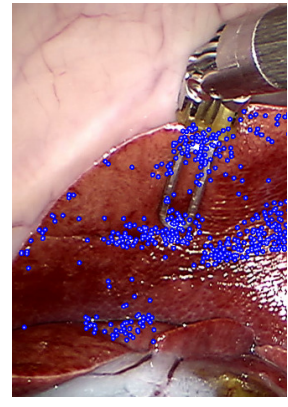

(a)

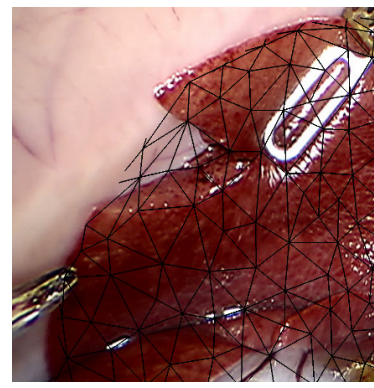

(e)

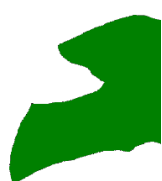

(h)

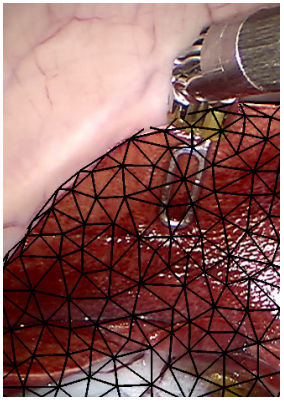

(b)

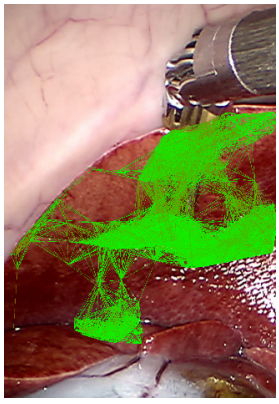

(c)

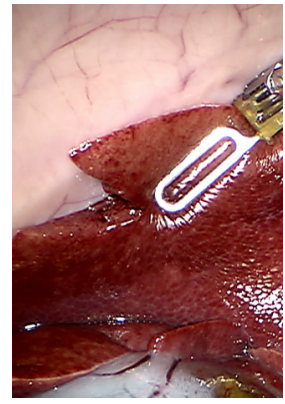

(d)

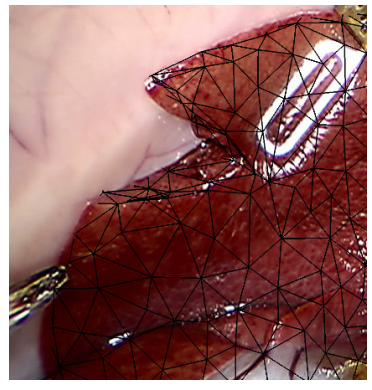

(f)

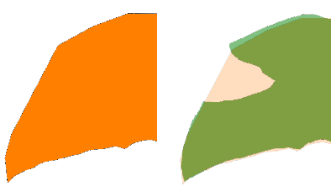

(i)

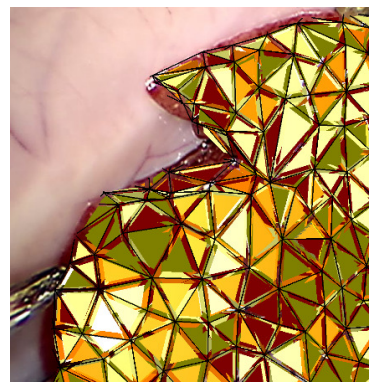

(g)

Fig. 4. (a) Detected features $f_{i}$; (b) FEM mesh of the virtual organ; (c) Computed neighborhood $\mathcal{N}$; (d) Organ manipulation after a cut; Augmented reality on cut and deformed liver without cut in the virtual organ (e) and with our method applying the cut to the virtual organ (f), (g); Surface areas of the real cut surface (h), models without/with cutting $(\mathrm{i}) /(\mathrm{j})$ 


\section{Conclusion and Discussion}

This work addresses the important and little studied problem of cutting during surgical augmented reality. The proposed method is able to detect a surgical cut applied on soft deformable structures, by analyzing the discontinuities in the motion of feature points obtained by visual tracking. The cut is applied on the preoperative model in real-time using an efficient combination of remeshing and snapping techniques, to maintain a realistic augmentation after a performed cut. Convincing preliminary results are demonstrated for both in vitro and in vivo examples. Let us point out that cuts can be detected even if the tracked features relatively far around the cut region, but with a deteriorated accuracy. More validation is obviously required though it is worth mentioning that validation implying actual organs are seldom reported in previous works. The current method is restricted to precut objects, the next steps in the context of this research will be an expansion to live cutting, tearing and fracture. For this step it is important to use less parameters or to automatically tune parameters to a specific scene. Beyond that - as our method does not address the depth of the cut - it would be particularly interesting to investigate organs partially cut in the direction of the camera.

\section{References}

1. Nicolau, S., Soler, L., Mutter, D., Marescaux, J.: Augmented reality in laparoscopic surgical oncology. Surgical Oncology 20(3) (2011)

2. Suwelack, S., et al.: Physics-based shape matching for intraoperative image guidance. Medical physics 41(11) (2014)

3. Haouchine, N., Dequidt, J., Peterlik, I., Kerrien, E., Berger, M.O., Cotin, S.: Imageguided simulation of heterogeneous tissue deformation for augmented reality during hepatic surgery. In: International Symposium on Mixed and Augmented Reality (ISMAR). (2013)

4. Pratt, P., Stoyanov, D., Visentini-Scarzanella, M., Yang, G.Z.: Dynamic guidance for robotic surgery using image-constrained biomechanical models. In: MICCAI. (2010)

5. Ferrant, M., Nabavi, A., Macq, B., Black, P., Jolesz, F., Kikinis, R., Warfield, S.: Serial registration of intraoperative $\mathrm{mr}$ images of the brain. Medical Image Analysis 6 (2001)

6. Wu, J., Westermann, R., Dick, C.: Physically-based simulation of cuts in deformable bodies: A survey. In: Eurographics 2014 State-of-the-Art Report. (2014)

7. Dick, C., Georgii, J., Westermann, R.: A hexahedral multigrid approach for simulating cuts in deformable objects. IEEE Transactions on Visualization and Computer Graphics 17(11) (2011)

8. Haouchine, N., Dequidt, J., Berger, M.O., Cotin, S.: Single View Augmentation of 3D Elastic Objects. In: ISMAR. (2014)

9. Koschier, D., Lipponer, S., Bender, J.: Adaptive tetrahedral meshes for brittle fracture simulation. In: Proceedings of the 2014 ACM SIGGRAPH/Eurographics Symposium on Computer Animation. (2014)

10. Nienhuys, H.W., Frank van der Stappen, A.: A surgery simulation supporting cuts and finite element deformation. In: MICCAI. (2001) 